\title{
The Independent Effects of Psychosocial Stressors on Subclinical Psychosis: Findings From the Multinational EU-GEI Study
}

Citation for published version (APA):

Pignon, B., Lajnef, M., Kirkbride, J. B., Peyre, H., Ferchiou, A., Richard, J. R., Baudin, G., Tosato, S., Jongsma, H., de Haan, L., Tarricone, I., Bernardo, M., Velthorst, E., Braca, M., Arango, C., Arrojo, M., Bobes, J., Del-Ben, C. M., Di Forti, M., ... Schurhoff, F. (2021). The Independent Effects of Psychosocial Stressors on Subclinical Psychosis: Findings From the Multinational EU-GEI Study. Schizophrenia Bulletin, 47(6), 1674-1684. https://doi.org/10.1093/schbul/sbab060

Document status and date:

Published: 01/11/2021

DOI:

10.1093/schbul/sbab060

Document Version:

Publisher's PDF, also known as Version of record

\section{Document license:}

Taverne

Please check the document version of this publication:

- A submitted manuscript is the version of the article upon submission and before peer-review. There can be important differences between the submitted version and the official published version of record.

People interested in the research are advised to contact the author for the final version of the publication, or visit the DOI to the publisher's website.

- The final author version and the galley proof are versions of the publication after peer review.

- The final published version features the final layout of the paper including the volume, issue and page numbers.

Link to publication

\footnotetext{
General rights rights.

- You may freely distribute the URL identifying the publication in the public portal. please follow below link for the End User Agreement:

www.umlib.nl/taverne-license

Take down policy

If you believe that this document breaches copyright please contact us at:

repository@maastrichtuniversity.nl

providing details and we will investigate your claim.
}

Copyright and moral rights for the publications made accessible in the public portal are retained by the authors and/or other copyright owners and it is a condition of accessing publications that users recognise and abide by the legal requirements associated with these

- Users may download and print one copy of any publication from the public portal for the purpose of private study or research.

- You may not further distribute the material or use it for any profit-making activity or commercial gain

If the publication is distributed under the terms of Article $25 \mathrm{fa}$ of the Dutch Copyright Act, indicated by the "Taverne" license above, 


\title{
The Independent Effects of Psychosocial Stressors on Subclinical Psychosis: Findings From the Multinational EU-GEI Study
}

\author{
Baptiste Pignon*,1, , Mohamed Lajnef ${ }^{1}$, James B. Kirkbride ${ }^{2, \bullet}$, Hugo Peyre ${ }^{3}$, Aziz Ferchiou1, Jean-Romain Richard ${ }^{1}$, \\ Grégoire Baudin $^{4}$, Sarah Tosato ${ }^{5}$, Hannah Jongsma ${ }^{6,7}$, Lieuwe de Haan ${ }^{8,9}$, Ilaria Tarricone ${ }^{10}$, Miguel Bernardo ${ }^{11}$, \\ Eva Velthorst ${ }^{12}$, Mauro Braca ${ }^{13}$, Celso Arango ${ }^{14}$, Manuel Arrojo ${ }^{15}$, Julio Bobes ${ }^{16}$, Cristina Marta Del-Ben ${ }^{17}$, \\ Marta Di Forti ${ }^{18,19}$, Charlotte Gayer-Anderson ${ }^{20}$, Peter B. Jones ${ }^{21,22}$, Caterina La Cascia ${ }^{23}$, Antonio Lasalvia, \\ Paulo Rossi Menezes ${ }^{24}$, Diego Quattrone ${ }^{25}$, Julio Sanjuán ${ }^{26,27,28}$, Jean-Paul Selten ${ }^{29,30}$, Andrea Tortelli31,32, \\ Pierre-Michel Llorca ${ }^{33,34}$, Jim van Os ${ }^{35,36,37}$, Bart P. F. Rutten ${ }^{38}$, Robin M. Murray ${ }^{39}$, Craig Morgan ${ }^{20}$, Marion Leboyer ${ }^{1}$, \\ Andrei Szöke ${ }^{1}$, and Franck Schürhoff ${ }^{1}$
}

${ }^{1}$ Univ Paris Est Creteil, INSERM, IMRB, AP-HP, Hôpitaux Universitaires « H. Mondor», DMU IMPACT, Fondation FondaMental, Créteil, France; ${ }^{2}$ PsyLife group, Division of Psychiatry, UCL, London, UK; ${ }^{3}$ AP-HP, Hôpital universitaire Robert Debré, Service de pédopsychiatrie, Paris, France; ${ }^{4}$ Université de Paris, Laboratoire de Psychopathologie et Processus de Santé, Boulogne Billancourt, France; ${ }^{5}$ Section of Psychiatry, Department of Neuroscience, Biomedicine and Movement Sciences, University of Verona, Verona, Italy; ${ }^{6}$ Centre for Transcultural Psychiatry "Veldzicht." Balkbrug, the Netherlands; VR Mental Health Group, University Center for Psychiatry, Univerisity Medical Centre Groningen, Groningen, The Netherlands; ${ }^{7}$ Centre for Longitudinal Studies, UCL, London, UK; ${ }^{8}$ Amsterdam UMC, Amsterdam, The Netherlands; ${ }^{9}$ Arkin, Amsterdam, The Netherlands; ${ }^{10}$ Department of Medical and Surgical Sciences, Bologna University, Bologna, Italy; ${ }^{11}$ Barcelona Clínic Schizophrenia Unit, Hospital Clínic of Barcelona, Institute of Neuroscience, University of Barcelona; Institut d'investigacions Biomèdiques August Pi i Sunyer (IDIBAPS), Barcelona; Centro de Investigación Biomédica en Red de Salud Mental (CIBERSAM), Spain; ${ }^{12}$ Department of Psychiatry, Icahn School of Medicine at Mount Sinai, New York, NY; ${ }^{13}$ Department of Mental Health and Pathological Addictions, Local Health Authority, Bologna, Italy; ${ }^{14}$ Department of Child and Adolescent Psychiatry, Institute of Psychiatry and Mental Health, Hospital General.Universitario Gregorio Marañón, Gregorio Marañón, (IiGSM), School of Medicine, Universidad Complutense de Madrid; CIBERSAM, Madrid, Spain; ${ }^{15}$ Department of Psychiatry, Psychiatric Genetic Group, Instituto de Investigación Sanitaria de Santiago de Compostela, Complejo Hospitalario Universitario de Santiago de Compostela, Spain; ${ }^{16}$ Faculty of Medicine and Health Sciences - Psychiatry, Universidad de Oviedo; ISPA, INEUROPA CIBERSAM, Oviedo, Spain; ${ }^{17}$ Department of Neuroscience and Behaviour, Ribeirão Preto Medical School, University of São Paulo, São Paulo, Brazil; ${ }^{18}$ Social, Genetic and Developmental Psychiatry Centre, Institute of Psychiatry, Psychology and Neuroscience, King's College London, London, UK; ${ }^{19}$ South London and Maudsley NHS Mental Health Foundation Trust, London, UK; ${ }^{20}$ Department of Health Service and Population Research, Institute of Psychiatry, King's College London, De Crespigny Park, Denmark Hill, London, UK; ${ }^{21}$ Department of Psychiatry, University of Cambridge, Cambridge, UK; ${ }^{22} \mathrm{CAMEO}$, Cambridgeshire \& Peterborough NHS Foundation Trust, Cambridge, UK; ${ }^{23}$ Department of Biomedicine, Neuroscience and Advanced Diagnostics, School of Medicine, University of Palermo, Palermo, Italy; ${ }^{24}$ Department of Preventive Medicine, Faculdade de Medicina, Universidade of São Paulo, São Paulo, Brazil; ${ }^{25}$ Social, Genetic, and Developmental Psychiatry Centre, Institute of Psychiatry, Psychology and Neuroscience, King's College London, London, UK; ${ }^{26}$ Biomedical Research Networking Centre in Mental Health (CIBERSAM), Madrid, Spain; ${ }^{27}$ Department of Psychiatry, Hospital Clínico Universitario de Valencia, School of Medicine, Universidad de Valencia, Valencia, Spain; ${ }^{28}$ Biomedical Research Institute INCLIVA, Valencia, Spain; ${ }^{29}$ Rivierduinen Institute for Mental Health, Leiden, The Netherlands; ${ }^{30}$ Maastricht University Medical Center, Departmentof Psychiatry \& Neuropsychology, School for Mental Health and Neuroscience, Maastricht, The Netherlands; ${ }^{31}$ French National Institute of Health and Medical Research (INSERM), Créteil, France; ${ }^{32} \mathrm{EPS}$ Maison Blanche, Paris, France; ${ }^{33} \mathrm{CHU}$ Clermont-Ferrand, Psychiatrie B, Clermont-Ferrand, France; ${ }^{34}$ Université Clermont Auvergne, Clermont-Ferrand, France; ${ }^{35}$ Department of Psychiatry and Neuropsychology, School for Mental Health and Neuroscience, Maastricht University Medical Centre, Maastricht, The Netherlands; ${ }^{36}$ Department of Psychiatry, UMC Utrecht Brain Centre, University Medical Centre Utrecht, Utrecht University, Utrecht, The Netherlands; ${ }^{37}$ Department of Psychosis Studies, Institute of Psychiatry, Psychology \& Neuroscience, King's College London, London, UK; ${ }^{38}$ Department of Psychiatry and Neuropsychology, School for Mental Health and Neuroscience, Maastricht University Medical Centre, Maastricht, The Netherlands; ${ }^{39}$ The Institute of Psychiatry, Psychology and Neuroscience, King's College London, London, UK

*To whom correspondence should be addressed; Hôpital Albert Chenevier, Groupe hospitalier Henri-Mondor, CHU de Créteil, Assistance Publique-Hôpitaux de Paris (AP-HP), 40 rue de Mesly, 94000 Créteil, France; tel: 33-1-49-81-31-31, fax: +33-1-49-81-30-59, e-mail: baptistepignon@yahoo.fr 
The influence of psychosocial stressors on psychosis risk has usually been studied in isolation and after the onset of the disorder, potentially ignoring important confounding relationships or the fact that some stressors that may be the consequence of the disorder rather than preexisting. The study of subclinical psychosis could help to address some of these issues. In this study, we investigated whether there was (i) an association between dimensions of subclinical psychosis and several psychosocial stressors including: childhood trauma, self-reported discrimination experiences, low social capital, and stressful life experiences, and (ii) any evidence of environment-environment (ExE) interactions between these factors. Data were drawn from the EUGEI study, in which healthy controls $(N=1497)$ and siblings of subjects with a psychotic disorder $(N=265)$ were included in six countries. The association between psychosocial stressors and subclinical psychosis dimensions (positive, negative and depressive dimension as measured by the Community Assessment of Psychic Experiences (CAPE) scale) and possible ExE interactions were assessed using linear regression models. After adjusting for sex, age, ethnicity, country, and control/sibling status, childhood trauma ( $\beta$ for positive dimension: 0.13 , negative: 0.49 , depressive: 0.26 ) and stressful life events (positive: 0.08 , negative: 0.16 , depressive: 0.17 ) were associated with the three dimensions. Lower social capital was associated with the negative and depression dimensions (negative: 0.26 , depressive: 0.13 ), and self-reported discrimination experiences with the positive dimension (0.06). Our findings are in favor of independent, cumulative and non-specific influences of social adversities in subclinical psychosis in non-clinical populations, without arguments for $\mathrm{E} \times \mathrm{E}$ interactions.

Key words: subclinical psychosis/schizotypy/psychotic symptoms/positive subclinical symptoms/negative subclinical symptoms/depressive subclinical symptoms/psychosocial stress/childhood trauma/stressful life events/social capital/dis crimination/Community Assessment of Psychic Experiences (CAPE)

\section{Introduction}

The stress-vulnerability theory of psychotic disorders posits that genetic factors interact with environmental stressors in the development of a disorder. ${ }^{1-3}$ In this theoretical model, increased sensitivity to stress plays an important role in both onset and relapse of psychotic disorders, including schizophrenia. Extending this model, the stress sensitization hypothesis proposes that repeated exposure to environmental stressors sensitizes key neurobiological pathways to psychosis. ${ }^{4,5}$ Early, severe or prolonged exposure to stress would lead to a dysregulated stress response and potentially explain both the role of early and current stress in the etiology of psychotic disorders.

Several environmental factors that could be conceptualized as psychosocial stressors, such as childhood trauma, stressful life events, discrimination experiences or a low level of social capital have been found to increase the risk of psychotic disorders. ${ }^{6-9}$ Furthermore, several studies support a stress sensitization model in psychosis, showing for example that exposure to an early stressor (childhood trauma) increases later sensitivity to other stressors (eg, social stress, population density, etc.) in patients (environment-environment ExE interaction). ${ }^{10,11}$ However, the fact that these studies have taken place after the onset of psychotic disorder raises causality concerns. Indeed, the assessment of recent stress could be confounded by several stressors associated with the disease itself, such as hospitalizations, stigma, substance use disorders or social drift after onset. ${ }^{12-14}$

Psychotic symptoms may be present, to varying degrees, in non-clinical, general population samples who do not meet criteria for a clinical disorder. ${ }^{15-17}$ The continuum theory of psychosis posits that subclinical experiences have a similar origin/etiology to full psychotic disorders. ${ }^{18-22}$ Thus, studying risk factors associated with subclinical psychosis may provide insights into the etiology of psychosis, while reducing the potential interference of reverse causation, that is, stress caused by the clinical disorders themselves. Furthermore, in line with the continuum theory, subclinical psychosis can be characterized by quantitative (continuous) variables, improving statistical power and the capacity to control for more potential confounders.

Several studies have previously reported associations between psychosocial stressors and subclinical psychosis. For instance, childhood trauma has been associated with increased rates of isolated psychotic symptoms in several studies. ${ }^{23-26}$ Likewise, negative and/or stressful life events $^{27,28}$ or self-reported discrimination experiences ${ }^{29}$ have also been associated with subclinical psychosis.

One major limitation of studies published to date is that psychosocial stressors have usually been studied in isolation, which might lead to spurious or incomplete conclusions given the likely presence of confounding/interaction with other stressors. Further work is needed to determine whether different psychosocial stressors have independent effects on subclinical psychosis and underlying dimensions (ie, positive, negative, depressive), while controlling for other relevant stressors. Moreover, the role of population-level (eg, social capital) factors that might interact with psychosocial stressors has rarely been explored. For example, social capital has been related to the incidence and outcome of psychotic disorders ${ }^{30,31}$ and low levels of social capital could be a stressful condition per $s e^{32}$ (ie, an independent stressor), or exacerbate the effects of other psychosocial factors (discrimination, trauma, stressful life events) on subsequent psychosis risk, but evidence is lacking. Relationships between social capital and subclinical psychosis have never been investigated. Moreover, to the best of our knowledge, only two studies have analyzed the role of multiple psychosocial stressors 
on subclinical psychosis. The first found arguments in favor of the sensitization hypothesis. ${ }^{33}$ The second, studying the role of childhood trauma alongside other environmental and genetic risk factors, found additive effects of these factors on subclinical psychosis scores. ${ }^{21}$

Furthermore, recent studies have reported that exposure to psychosocial stressors was in part dependent on genetic vulnerability to psychosis, ${ }^{34,35}$ and no study has compared associations between psychosocial stressors and subclinical psychosis in both control participants and (healthy) siblings of people with a psychotic disorder. Including both groups may help tease out the genetic and environmental etiology of psychosis.

To address some of the limitations of previous work, we used a large, cross-national sample of populationbased controls and siblings of subjects with a psychotic disorder, to investigate whether there was (1) an association between subclinical psychosis dimensions (positive, negative and depressive) and psychosocial stressors, and (2) any evidence of ExE interactions between different psychosocial stressors in line with the stress sensitization hypothesis. The psychosocial stressors we explored can be conceptualized as either "early" (childhood trauma) or "prolonged" (discrimination, low level of social capital, stressful life experiences). We hypothesized that exposure to "early" stressors would enhance the effect of adversity later in life.

\section{Methods}

\section{EU-GEI Study}

Data were collected in the "European network of national schizophrenia networks studying gene-environment interactions" (EU-GEI) study, a multicentre case-siblingcontrol study of genetic and environmental determinants of the occurrence, severity and outcome of psychotic disorders. For the second work-package of the study (WP2: Functional Enviromics), three categories of participants were recruited between 2010 and 2015: (1) subjects presenting with a first-episode of psychotic disorder (FEP), (2) population-based healthy controls, and; (3) siblings of participants with FEP. ${ }^{36}$ Participants were recruited across six countries: Brazil, France, Italy, the Netherlands, Spain, and the United Kingdom. ${ }^{37,38}$ In the present analyses, only controls and siblings were included.

\section{Subjects}

Population-based controls and siblings had no personal history of psychosis, and controls were recruited from the same catchment areas as the cases. In each centre, controls were recruited using a mixture of random and quota sampling to ensure control participants were broadly representative of the population at-risk from which cases could present in each catchment area on predefined variables (age, sex, and migration). ${ }^{36}$

\section{Subclinical Psychosis Measure}

The Community Assessment of Psychic Experiences (CAPE) is a 42-item, self-report questionnaire that has been developed to measure lifetime subclinical psychotic dimensions in the general population. ${ }^{39}$ For each item, four answers were possible according to the frequency of their occurrences (from never to nearly always). A meta-analysis found that the CAPE displays a three-dimensional symptom structure: positive, negative and depressive dimensions. ${ }^{40}$ We therefore used the sum of endorsed items to quantify each of the three psychotic dimensions consistent with previous studies. ${ }^{22,41,42}$ To construct these dimension scores, we dichotomized answers on each CAPE item (never vs. sometimes or more) and summed the positive answers. This led to theoretical positive dimension scores between 0 and 20, negative dimension scores between 0 and 14, and depressive dimension scores between 0 and 8 .

We have previously demonstrated the cross-national invariance of the CAPE assessment in the EUGEI WP2 samples: equivalent factorial structures, factor loadings and thresholds across the six countries. ${ }^{43}$ Thus, CAPE results can be reliably used across the different EUGEI countries.

\section{Psychosocial Stressors Measure}

Childhood trauma was assessed with the Childhood Trauma Questionnaire (CTQ), a 25-item questionnaire, that measures five different domains (emotional and physical neglect; emotional, physical and sexual abuse). All items are scored on a five-point Likert-scale (1: never, to 5: very often). We used the CTQ total score as the sum of all items, thus theoretically ranging from 25 to $125 .^{44}$

Lifetime self-reported discrimination experiences were assessed with a modified version of the Williams' major experiences of discrimination measure (available in Supplementary Material 1), a 12-item scale assessing several experiences of discrimination (unfairly fired or not hired because of your ethnicity/sex/weight/etc., unfairly stopped/questioned/physically threatened or abused by the police, etc.). ${ }^{45,46}$ This version of the Williams' scale has already been used in a paper studying the prevalence of discrimination in South London, and its relationships with psychiatric disorders. ${ }^{47}$ We used a total discrimination score by adding all endorsed items, ranging theoretically from 0 to 12 .

Perceived social capital in each participant's immediate neighborhood was assessed using the Social Environment Assessment Tool (SEAT), a 23-item questionnaire. This tool was designed to capture four dimensions of social capital: civic disorder (CD), impact of civic disorder (ICD), informal social control (ISC), and social cohesion and trust (SCT) ${ }^{48-51}$ Respondents answer according to a five-point Likert-scale (1: unusual, to 5: very common). Sum scores for 4 subscales 
were derived then standardized to $Z$-scores (ie, to a mean equal to 0 , and a standard-deviation equal to 1). The total social capital score was obtained by adding the weighted scale scores (SEAT score $=\mathrm{zCD}$ $+0.51{ }^{*} \mathrm{zICD}+1.6^{*} \mathrm{zISC}+\mathrm{zSCT}$ ) based on the factorial structure of the instrument. This scale has shown excellent goodness-of-fit statistics (data available on request forthcoming). Our analyses were restricted to the total social capital score, which we inverted for analyses so that higher scores were associated with lower social capital.

Finally, stressful life events were assessed using the List of Threatening Experiences (LTE) which comprises 20 binary items. ${ }^{52,53}$ This scale assesses 20 events usually associated with major stress over the course of the previous 6 months including: serious injury or illness in oneself or a close relative, death of parent/child/partner, death of a family member, death of a friend, separation from a partner, loss of job or financial difficulties. The total score ranges theoretically from 0 to 20 .

\section{Other Adjustment Variables}

We also collected information on age, sex, country, and ethnicity as possible confounding factors. Age was measured at the time of the interview. Ethnicity was selfdefined according to one of six categories: Asian, Black, North African, White, Mixed, Other.

\section{Ethical Procedures}

Ethical approval was obtained from local research ethics committees in each country. The EU-GEI Project was funded by the European Community's Seventh Framework Program under grant agreement no. HEALTH-F2-2010-241909.

\section{Statistical Methods}

First, we assessed the associations between psychosocial stressors using Spearman correlation tests. Then we analyzed the relationships between these stressors and the three CAPE dimensions scores also using Spearman correlation tests. We used Mann-Whitney $U$ tests to assess the differences in exposure to psychosocial stressors between controls and siblings.

Second, we fitted linear regression models to analyze independent and specific effects of each psychosocial stressor on each of our three subclinical psychosis outcomes (ie, each of the three CAPE dimensions). We fitted multivariable models, controlling for other relevant psychosocial stressors as well as age, sex, ethnicity, country, and control-sibling status (ie, a priori confounders). ${ }^{18,54,55}$ As CAPE scores did not follow a normal distribution (as shown by Shapiro tests with a $P$-value $<.05$, and graphical methods), a Box-Cox transformation of all continuous variables (CAPE scores) was performed to fulfil the normality assumption required by the parametric procedure. Complete case analysis was used.

Third, we tested for evidence of ExE interactions between our four psychosocial stressors on each outcome in our multivariable models. Each interaction (ie, CTQ $\times$ LTE, CTQ $\times$ SEAT, LTE $\times$ SEAT, etc.) was tested separately, by introducing interaction terms in the four multivariable models. In a final model, all the interaction terms were pooled into a single model for each of the outcomes.

To facilitate comparisons of effect sizes of the different psychosocial stressor measures, $Z$-scores of these variables were calculated and used in the multivariable models.

The analyses were repeated among sibling and control subsamples, as siblings may experience different exposure and different response to the same exposure.

Significance was based on a two-sided $P$-value of .05 throughout. R software version 3.6.0, with "stats," "car" and "lattice" packages, was used.

\section{Results}

\section{Sample Characteristics}

The sample was composed of 1762 subjects, including 1497 controls $(85.0 \%)$ and 265 siblings $(15.0 \%)$, of those 972 were men $(55.2 \%)$ and 790 were women $(44.8 \%)$. The median age for the controls was 33 years (IQR [26-47]), and 30 years (IQR [23-38]) for the siblings. The proportion of women was higher among controls, and controls were older. The proportion of people from nonwhite ethnic backgrounds differed between controls (21.3\%) and siblings (24.1\%), with a higher proportion of subjects of Black ethnicity ( $8.1 \%$ vs $4.9 \%$ ) and a lower proportion of subjects of Mixed ethnicity in controls $(7.7 \%$ vs $15.5 \%$ ). Regarding CAPE scores, positive dimensions were higher among controls in comparison with siblings. Scores on the CTQ were higher among siblings while siblings reported fewer discrimination experiences. More details are available in the table 1.

\section{Correlations Between Psychosocial Stressors}

The correlation matrix (table 2 ) revealed small but statistically robust $(P<.001)$ correlations between all stressors. We observed positive correlations between childhood trauma, self-reported discrimination experiences, and stressful life events (rho between 0.14 and 0.20), and negative correlations between high level of social capital and the three psychosocial stressors (rho between -0.15 and -0.08 ). Correlations between psychosocial stressors showed similar patterns of magnitude and direction within both the control and sibling groups (except for social capital and stressful life events and social capital among siblings which were not associated, see Supplementary table 2 for analyses within controls and siblings). 


\section{B. Pignon et al}

Table 1. Description and Comparisons of the CAPE and of the Psychosocial Stress Measures According to Control/Sibling Status

\begin{tabular}{|c|c|c|c|}
\hline & \multirow{2}{*}{$\begin{array}{l}\text { Controls }(N=1497) \\
\text { Median }(\mathrm{IQR}), \text { Mean }(\mathrm{SD}) \\
\text { or } \\
N(\%)\end{array}$} & \multirow{2}{*}{$\begin{array}{l}\text { Siblings }(N=265) \\
\text { Median }(\mathrm{IQR}), \text { Mean }(\mathrm{SD}) \\
\text { or } \\
N(\%)\end{array}$} & \multirow[t]{2}{*}{ Comparisons } \\
\hline & & & \\
\hline Age & $33(21), 36.1(12.9)$ & $30(15), 31.3(9.4), 1.9 \%$ & $<.01^{* *}$ \\
\hline \multicolumn{4}{|c|}{ סקום } \\
\hline Women & $791(47.2 \%)$ & $181(31.7 \%)$ & \multirow[t]{2}{*}{$<.01 *$} \\
\hline Men & $706(52.8 \%)$ & $84(68.3 \%)$ & \\
\hline \multicolumn{4}{|l|}{ Ethnicity } \\
\hline Asian & $33(2.2 \%)$ & $3(1.1 \%)$ & \multirow[t]{6}{*}{$<.01 *$} \\
\hline Black & $121(8.1 \%)$ & $13(4.9 \%)$ & \\
\hline North African & $24(1.6 \%)$ & $6(2.3 \%)$ & \\
\hline White & $1178(78.7 \%)$ & $201(75.9 \%)$ & \\
\hline Mixed & $116(7.7 \%)$ & $41(15.5 \%)$ & \\
\hline Other & $24(1.7 \%$ & $1(3.8 \%)$ & \\
\hline \multicolumn{4}{|l|}{$C A P E$ scores } \\
\hline Positive & $4(4), 4.9(2.9)$ & $4(4), 4.5(3.0)$ & $.02^{* *}$ \\
\hline Negative & $6(6), 6.2(3.6)$ & $6(6), 5.8(3.7)$ & $.18^{* *}$ \\
\hline Depressive & $4(3), 4.4(2.0)$ & $4(3), 4.2(2.1)$ & $.13^{* *}$ \\
\hline \multicolumn{4}{|l|}{ Psychosocial stressors } \\
\hline Childhood trauma & $31(11), 34.6(10.9)$ & $33(12), 37.3(6.9)$ & $<.01^{* *}$ \\
\hline Self-reported discrimination experiences & $0.0(1.0), 0.6(1.0)$ & $0.0(1.0), 0.4(0.9)$ & $.02^{* *}$ \\
\hline Stressful life events & $1(2), 1.5(1.4)$ & $1(1), 1.5(1.5)$ & $.29^{* *}$ \\
\hline Social capital & $0.0(3.4), 0(2.5)$ & $0.1(3.4), 0(2.5)$ & $.56^{* *}$ \\
\hline
\end{tabular}

Abbreviations: IQR, interquartile range; NA, unavailable data; SD, standard-deviation.

$* P$-value of chi-square tests.

** $P$-value of Mann-Whitney.

Table 2. Spearman Correlation Matrix of the Psychosocial Stressors

\begin{tabular}{|c|c|c|c|c|}
\hline & Childhood Trauma & $\begin{array}{l}\text { Self-Reported Discrimination } \\
\text { Experiences }\end{array}$ & Stressful Life Events & Social Capital \\
\hline Childhood trauma & 1.00 & & & \\
\hline $\begin{array}{l}\text { Self-reported discrimination ex- } \\
\text { periences }\end{array}$ & $0.17 * * *$ & 1.00 & & \\
\hline Stressful life events & $0.14 * * *$ & $0.20 * * *$ & 1.00 & \\
\hline Social capital & $-0.15 * * *$ & $-0.08 * * *$ & $-0.11 * * *$ & 1.00 \\
\hline
\end{tabular}

$P$-values: $*<0.05, * *<0.01, * * *<0.001$

\section{Correlations Between Subclinical Psychosis Scores and} Psychosocial Stressors

All psychosocial stressors were positively correlated with the different CAPE dimensions (table 3). Higher perceived social capital scores were associated, as expected, with lower positive, negative and depressive dimension scores. Correlations ranged from -0.13 (higher social capital associated with lower depressive score) to 0.29 (childhood trauma and negative dimension). Correlations showed similar patterns of direction within both the control and sibling groups, with higher levels of correlation between CAPE scores and self-reported discrimination experiences among siblings vs controls (see Supplementary table 3 for analyses within controls and siblings).

\section{Multivariable Modeling}

After adjusting for sex, age, ethnicity, country, and control/sibling status, the different CAPE scores were independently associated with childhood trauma $(\beta$ with $Z$-score of childhood trauma between 0.13 and 0.49 ) and stressful life events ( $\beta$ between 0.08 and 0.17 ). Lower social capital was associated with negative and depressive dimensions ( $\beta$ between 0.05 and 0.24 ), while self-reported discrimination experiences were only associated with the positive dimension $(\beta=0.06)$. None of the interaction terms of the psychosocial stressor measures (both when introduced one by one in the multivariable analyses, and in the models with all the interactions terms) were associated with any of the three dimensions (see Supplementary table 4 for the 
results of the interactions). Of note, sibling status was associated with lower scores on the positive dimension $(\beta=-0.16)$. The detailed results of the multivariable analyses with $Z$-scores of the psychosocial measures are available in the table 4.

The same multivariable analyses were repeated separately for siblings and controls, and revealed globally similar results. Of note, among siblings, fewer associations reached statistical significance, and the interaction between childhood trauma and discrimination was significant (negative interaction: $\beta=-0.15$, more details of the multivariable analyses within controls and siblings in Supplementary table 5).

\section{Discussion}

In the cross-national and non-clinical EUGEI sample we assessed the effects of several psychosocial stressors (childhood trauma, stressful life-events, self-reported discrimination experiences and low social capital) on different subclinical psychosis dimensions for the first time. Subclinical psychosis was assessed with the CAPE and all analyses were adjusted for relevant sociodemographic factors (age, sex, country and ethnicity). This revealed that childhood trauma and stressful life events were associated with higher scores on the positive, negative and depressive dimensions. Lower social capital was associated with higher scores on negative and depressive dimensions,

Table 3. Spearman Correlations Between CAPE Scores and Psychosocial Atressors

\begin{tabular}{lccc}
\hline & $\begin{array}{c}\text { Positive } \\
\text { Dimension* }\end{array}$ & $\begin{array}{c}\text { Negative } \\
\text { Dimension* }\end{array}$ & $\begin{array}{c}\text { Depressive } \\
\text { Dimension* }\end{array}$ \\
\hline $\begin{array}{l}\text { Childhood trauma } \\
\begin{array}{l}\text { Self-reported } \\
\text { discrimination }\end{array}\end{array}$ & 0.26 & 0.29 & 0.27 \\
$\begin{array}{l}\text { experiences } \\
\text { Stressful life events }\end{array}$ & 0.15 & 0.13 & 0.12 \\
Social capital & 0.18 & & \\
\hline
\end{tabular}

*All $P$-values $<0.001$. while self-reported discrimination experiences were associated with the positive dimension.

Overall, as all evaluated psychosocial stressors were associated with subclinical psychosis, and as no interaction between these stressors was significant, these findings are consistent with an independent effect of the different psychosocial stressors. Moreover, as the different psychosocial stressors were (with the exception of discrimination) similarly associated with the different dimensions, our findings are consistent with a common etiology for the three dimensions.

This study contributes to our understanding of the relationships between psychosis and early, recent, and prolonged psychosocial stressors. The major strength of this study is the concomitant analysis of several psychosocial stressors. In concordance with a recent study in the American general population, ${ }^{56}$ we confirm the existence of significant correlations between different stressors and we show that these stressors have independent effects on the three dimensions of subclinical psychosis. In addition, we found that a low level of social capital is associated with higher levels of negative and depressive dimensions. This ecological neighborhood-level factor had never been studied in relation with subclinical psychosis outcomes in adults before (of note, Solmi et al. found an association between maternal neighborhood stress and the rate of psychotic symptoms among 13-year-old adolescents ${ }^{32}$ ), while previous studies found associations with the incidence of psychotic disorders. ${ }^{30}$ This result is consistent with other studies regarding the influence of neighborhood characteristics such as as deprivation or social fragmentation on psychosis outcomes, ${ }^{6}$ including subclinical psychosis..$^{57,58}$

Surprisingly, the absence of any strong evidence of interactions between the psychosocial stressors and especially between early and recent stress was not consistent with our hypothesis and the sensitization hypothesis. This result also differs from the results of Lataster et al. ${ }^{33}$ In this 10-year follow-up of adolescents and young adults from the general population in Germany $(N=1722)$, authors found non-additive interactions

Table 4. Multivariable Analyses of the Relationships Between the Z-scores of the Different Psychosocial Stressors and the ThreeDimension Scores

\begin{tabular}{|c|c|c|c|c|c|c|}
\hline & \multicolumn{2}{|c|}{ Positive Dimension } & \multicolumn{2}{|c|}{ Negative Dimension } & \multicolumn{2}{|c|}{ Depressive Dimension } \\
\hline & $\beta$ & $95 \% \mathrm{CI}$ & $\beta$ & $95 \% \mathrm{CI}$ & $\beta$ & $95 \% \mathrm{CI}$ \\
\hline Childhood trauma & 0.13 & {$[0.10 ; 0.17]^{* * *}$} & 0.49 & {$[0.37 ; 0.61]^{* * *}$} & 0.26 & {$[0.19 ; 0.33]^{* * *}$} \\
\hline Self-reported discrimination experiences & 0.06 & {$[0.02 ; 0.10]^{* *}$} & 0.05 & {$[-0.09 ; 0.19]$} & 0.05 & {$[-0.03 ; 0.13]$} \\
\hline Stressful life events & 0.08 & {$[0.04 ; 0.12]^{* * *}$} & 0.16 & {$[0.04 ; 0.28]^{*}$} & 0.17 & {$[0.11 ; 0.24]^{* * *}$} \\
\hline Lower social capital & 0.03 & {$[0.00 ; 0.07]$} & 0.26 & {$[0.14 ; 0.38]^{* * *}$} & 0.13 & {$[0.06 ; 0.20]^{* * *}$} \\
\hline Siblings (vs. controls) & -0.16 & {$[-0.26 ;-0.05]^{* *}$} & -0.28 & {$[-0.62 ;-0.06]$} & -0.19 & {$[-0.19 ; 0.01]$} \\
\hline
\end{tabular}

${ }^{a}$ Adjusted for age, sex, country, and ethnicity.

$*<.05, * *<.01, * * *<0001$. 
between early and recent psychosocial risk factors on the risk for positive psychotic symptoms (ie, delusional symptoms and/or hallucinations): unadjusted analyses showed associations between early and recent adversity and further psychotic symptoms, but these associations disappeared after statistical adjustment. Additive interactions between early and recent adversity were significant only for the fourth and highest level of recent adversity (adjusted risk ratio for the combined early and higher level of recent adversity $=4.08,95 \%$ CI $[2.02$ $8.24], P$-value $=.032$ ). Differences in results of this and our study might be explained by important methodological differences. For example, Lataster et al. study was longitudinal (three follow-up surveys covering a mean period of 8.4 years) and analyzed only two psychosocial stressors categorical variables (childhood trauma, dichotomized; and recent trauma and negative life events, four levels).

Analyzing the influence of environmental (including childhood trauma, cannabis use and urbanicity), sensory (hearing impairment), and familial risk factors on the occurrence of subclinical psychosis in 6646 subjects from the general population, Pries et al. found additive effects of the risk factors: the greater the number of risk factors, the greater the odds of symptoms..$^{21}$ Consistent with this finding, in our study, the effect of recent adverse experiences (measured by the LTE) was significant for each of the three dimensions, independently of early adverse experience (measured by childhood trauma), and we did not find any significant interactions between the different psychosocial stressors. This may be due to a lack of statistical power to detect an ExE interaction, ${ }^{59}$ however the $\beta$ values of the interactions, which are close to 0 make this unlikely.

Childhood trauma, that is, the earliest psychosocial stressor, had the strongest associations with each of the three dimensions measured. Childhood adverse experiences have been linked with long-term changes in the hypothalamic-pituitary axis (HPA) axis ${ }^{60}$ which may be involved in early stressor and emotional dysregulation, leading to later aberrant salience, involved in the positive dimension of psychosis. ${ }^{61}$ Of note, childhood trauma is known to be associated with psychopathology as a whole, ${ }^{62,63}$ and consistent with prior research, childhood trauma in our study was also associated with the negative dimension ${ }^{64}$ as well as with depressive symptoms ${ }^{65}$ in both controls and siblings.

Self-reported discrimination experiences were associated with the positive dimension of psychosis in the global sample. Several studies, including longitudinal studies, ${ }^{66}$ have shown associations, particularly with psychotic-like experiences. They have been confirmed in recent metaanalyses. ${ }^{9,29}$ Consistently with the present study, some studies suggest that among the different dimensions of subclinical psychosis discrimination is specifically associated with positive psychotic symptoms. ${ }^{9,67}$ However, in contrast with the present study, discrimination experiences have also been linked to depression. ${ }^{68}$

The associations between CAPE scores and stressful life events support the role of recent adverse experiences in the development of psychosis. ${ }^{8,61}$ Psychosis has been theorized to combine cognitive (external attribution) bias and emotional dysregulation. ${ }^{69}$ Stressful life events could play a trigger role for a disruption in cognitive processes in subjects at risk, leading to delusional ideas and hallucinatory experiences, the content of which may be influenced by the emotional change induced by the stressful events. $^{70}$

One point that also deserves discussion is the lower levels of the positive dimension of subclinical psychosis among siblings in comparison to controls. This result is surprising, as siblings of people with psychotic disorder face both a greater genetic predisposition to psychotic disorders, ${ }^{71,72}$ and higher levels of psychosocial stressors, because they likely share some of the environmental characteristics of their sibling (eg, discrimination), ${ }^{73,74}$ and the stress associated with the psychiatric disorder of their sibling. ${ }^{75}$ Indeed, consistent with the stress-vulnerability hypothesis, several studies found higher levels of subclinical psychosis among siblings, in comparison to controls. ${ }^{64,74,76}$ One hypothesis that might explain the negative association in our study is that siblings might minimize their symptoms, either because they compare them to the symptoms of their sibling with a FEP, or because they fear having the same disorder, and thus deny presenting the same symptoms. Moreover, we cannot exclude a selection bias, which may have occurred if siblings with potentially higher subclinical psychosis scores refused participation. Nevertheless, in our study, the stronger association between CAPE scores and self-reported discrimination in siblings is consistent with the stress-vulnerability hypothesis. Indeed, in siblings who share the genetic liability to psychosis (ie, a vulnerability) levels of self-reported discrimination experiences are lower than in controls, but their effect is stronger. A similar result has been shown concerning urbanicity, which has been associated with a higher risk for psychotic disorders among subjects with familial liability as compared to subjects without. ${ }^{77}$

Several limitations should be acknowledged. First, we performed cross-sectional analyses of both subclinical psychosis and psychosocial stressors with retrospective assessment for some of the variables. Due to the retrospective assessment, recall bias and reverse causality cannot be excluded entirely. Indeed, several studies showed discrepancies between prospective and retrospective measures. ${ }^{78}$ Retrospective assessment (eg, for childhood trauma) may be biased by clinical outcomes (ie, reverse causation issue) ${ }^{79}$ for instance. Similarly, the perception of discrimination could be distorted by the presence of positive symptoms. Likewise, the perception of a low level of social capital could be influenced by depressive symptoms. With regard to the measure 
of stressful life events specifically, several issues have been mentioned, including intra-category variability (ie, the fact that subjects have differing views of what comprises a "major" or "serious" event or disease), and that this variability could also be related to psychiatric symptoms. ${ }^{53,80}$ Moreover, the mean discrimination score (median of discrimination measure: $0, \mathrm{IQR}=1$ ) was quite low in comparison with other studies (which unlike our study, were conducted among ethnic minorities facing higher levels of discrimination ${ }^{29,81,82}$ ). This low score has been occasionally found in previous studies, ${ }^{66,83}$ but should be interpreted taking into consideration that the version of the Williams' Major experiences of discrimination measure ${ }^{46,47}$ has not been validated, and its cross-national invariance has not yet been studied. Thus, the findings may not be generalizable to other countries (eg, USA). Moreover, the view of discrimination as a prolonged stressor might be misconstrued, as a major part of the experiences measured occur during adulthood. Certain experiences may however take place during childhood, adolescence or youth (unfair treatment when seeking medical care, discouragement from continuing education, etc.). Furthermore, except for ethnicity, the analyses were not adjusted for other important risk factors of psychosis, such as urbanicity and cannabis use ${ }^{84,85}$ However, adjustment for all the known risk factors of psychosis (economic deprivation, obstetrical complications, paternal age, etc.) was not possible, and such adjustment could cause statistical overadjustment and affect genuine relationships between subclinical psychosis and psychosocial stressors. Finally, as the sampling was not fully at random, but a mixture of random and quota sampling and thus non-probabilistic, we cannot assume that our sample was representative of the general population. However, the quota sampling method warrant same socio-demographic characteristics of the general population (age, sex, and migration) in the different countries. Moreover, we cannot exclude the possibility of selective refusal of study inclusion according to subclinical psychosis and/or psychosocial factors.

Overall, this international and multicentre study assessed positive, negative and depressive dimensions of subclinical psychosis among controls and siblings from the general population and simultaneously analyzed the role of different psychosocial stressors. Childhood trauma, and stressful life events were significantly associated with the three subclinical psychosis dimensions, while lower social capital was associated with the negative and depressive dimensions, and self-reported discrimination experiences associated with the positive dimension, consistent with independent effects of these different psychosocial stressors.

\section{Acknowledgments}

EU-GEI WP2 Group: Kathryn Hubbard, Stephanie Beards, Simona A. Stilo, Mara Parellada, Pedro Cuadrado, José Juan Rodríguez Solano, Angel Carracedo, David Fraguas, Álvaro Andreu-Bernabeu, Gonzalo López, Bibiana Cabrera, Esther Lorente-Rovira, Paz GarciaPortilla, Javier Costas, Estela Jiménez-López, Mario Matteis, Marta Rapado-Castro, Emiliano González, Covadonga M. Díaz-Caneja, Emilio Sánchez, Manuel Durán-Cutilla, Nathalie Franke, Fabian Termorshuizen, Daniella van Dam, Elsje van der Ven, Elles Messchaart, Stéphane Jamain, Thomas Charpeaud, Anne-Marie Tronche, Flora Frijda, Giovanna Marrazzo, Lucia Sideli, Crocettarachele Sartorio, Fabio Seminerio, Camila Marcelino Loureiro, Rosana Shuhama, Mirella Ruggeri, Chiara Bonetto, Doriana Cristofalo, Domnico Berardi, Marco Seri, Elena Bonora, Giuseppe D’Andrea, Laura Ferraro, Giada Tripoli, Silvia Amoretti, Gisela Mezquida

\section{Conflicts of Interest}

The funder had no involvement in study design, data collection, analysis, interpretation of findings, manuscript preparation or the decision to submit the paper for publication.

Dr Kirkbride is supported by the National Institute for Health Research (NIHR) University College London Hospital (UCLH) Biomedical Research Centre (BRC).

Dr Bernardo has been a consultant for, received grant/ research support and honoraria from, and been on the speakers/advisory board of ABBiotics, Adamed, Angelini, Casen Recordati, Janssen-Cilag, Menarini, Rovi and Takeda.

Dr Arango has received support by the Spanish Ministry of Science and Innovation. Instituto de Salud Carlos III (SAM16PE07CP1, PI16/02012, PI19/024), co-financed by ERDF Funds from the European Commission, "A way of making Europe," CIBERSAM. Madrid Regional Government (B2017/BMD-3740 AGES-CM-2), European Union Structural Funds. European Union Seventh Framework Program under grant agreements FP7-4-HEALTH-2009-2.2.1-2-241909 (Project EU-GEI) and FP7-HEALTH-2013-2.2.1-2-603196 (Project PSYSCAN); and European Union H2020 Program under the Innovative Medicines Initiative 2 Joint Undertaking (grant agreement No 115916, Project PRISM, and grant agreement No 777394, Project AIMS2-TRIALS), Fundación Familia Alonso and Fundación Alicia Koplowitz.

Dr Di Forti reports personal fees from Janssen outside the submitted work, without conflict of interest with the present study. 


\section{References}

1. Corcoran C, Walker E, Huot R, et al. The stress cascade and schizophrenia: etiology and onset. Schizophr Bull. 2003;29(4):671-692.

2. van Os J, Kenis G, Rutten BP. The environment and schizophrenia. Nature. 2010;468(7321):203-212.

3. Davis J, Eyre H, Jacka FN, et al. A review of vulnerability and risks for schizophrenia: beyond the two hit hypothesis. Neurosci Biobehav Rev. 2016;65:185-194.

4. Howes OD, Murray RM. Schizophrenia: an integrated sociodevelopmental-cognitive model. Lancet. 2014;383(9929):1677-1687.

5. Weidenauer A, Bauer M, Sauerzopf U, et al. Making sense of: sensitization in schizophrenia. Int J Neuropsychopharmacol. 2017;20(1):1-10.

6. March D, Hatch SL, Morgan C, et al. Psychosis and place. Epidemiol Rev. 2008;30:84-100.

7. Varese F, Smeets F, Drukker M, et al. Childhood adversities increase the risk of psychosis: a meta-analysis of patientcontrol, prospective- and cross-sectional cohort studies. Schizophr Bull. 2012;38(4):661-671.

8. Beards S, Gayer-Anderson C, Borges S, Dewey ME, Fisher HL, Morgan C. Life events and psychosis: a review and meta-analysis. Schizophr Bull. 2013;39(4):740-747.

9. Pearce J, Rafiq S, Simpson J, Varese F. Perceived discrimination and psychosis: a systematic review of the literature. Soc Psychiatry Psychiatr Epidemiol. 2019;54(9):1023-1044.

10. Lardinois M, Lataster T, Mengelers R, Van Os J, MyinGermeys I. Childhood trauma and increased stress sensitivity in psychosis. Acta Psychiatr Scand. 2011;123(1):28-35.

11. Veling W, Pot-Kolder R, Counotte J, van Os J, van der Gaag M. Environmental social stress, paranoia and psychosis liability: a virtual reality study. Schizophr Bull. 2016;42(6):1363-1371.

12. Zipursky RB. Why are the outcomes in patients with schizophrenia so poor? J Clin Psychiatry. 2014;75(Suppl 2):20-24.

13. Sariaslan A, Fazel S, D'Onofrio BM, et al. Schizophrenia and subsequent neighborhood deprivation: revisiting the social drift hypothesis using population, twin and molecular genetic data. Transl Psychiatry. 2016;6:e796.

14. Pignon B, Eaton S, Schürhoff F, Szöke A, McGorry P, O'Donoghue B. Residential social drift in the two years following a first episode of psychosis. Schizophr Res. 2019;210:323-325.

15. Verdoux H, van Os J. Psychotic symptoms in non-clinical populations and the continuum of psychosis. Schizophr Res. 2002;54(1-2):59-65.

16. McGrath JJ, Saha S, Al-Hamzawi A, et al. Psychotic experiences in the general population: a cross-national analysis based on 31,261 respondents from 18 countries. JAMA Psychiatry. 2015;72(7):697-705.

17. Pignon B, Schürhoff F, Szöke A, et al. Sociodemographic and clinical correlates of psychotic symptoms in the general population: findings from the MHGP survey. Schizophr Res. 2018;193:336-342.

18. Linscott RJ, van Os J. An updated and conservative systematic review and meta-analysis of epidemiological evidence on psychotic experiences in children and adults: on the pathway from proneness to persistence to dimensional expression across mental disorders. Psychol Med. 2013;43(6):1133-1149.

19. Szöke A, Kirkbride JB, Schürhoff F. Universal prevention of schizophrenia and surrogate endpoints at population level. Soc Psychiatry Psychiatr Epidemiol. 2014;49(9):1347-1351.
20. van Os J. The many continua of psychosis. JAMA Psychiatry. 2014;71(9):985-986.

21. Pries LK, Guloksuz S, Ten Have M, et al. Evidence that environmental and familial risks for psychosis additively impact a multidimensional subthreshold psychosis syndrome. Schizophr Bull. 2018;44(4):710-719.

22. Schürhoff F, Pignon B, Lajnef M, et al. Psychotic experiences are associated with paternal age but not with delayed fatherhood in a large, multinational, community sample. Schizophr Bull. 2020;46(5):1327-1334.

23. Cougnard A, Marcelis M, Myin-Germeys I, et al. Does normal developmental expression of psychosis combine with environmental risk to cause persistence of psychosis? A psychosis proneness-persistence model. Psychol Med. 2007;37(4):513-527.

24. Morgan C, Reininghaus U, Reichenberg A, Frissa S, Hotopf M, Hatch SL; SELCoH study team. Adversity, cannabis use and psychotic experiences: evidence of cumulative and synergistic effects. Br J Psychiatry. 2014;204:346-353.

25. McGrath JJ, Saha S, Lim CCW, et al.; WHO World Mental Health Survey Collaborators. Trauma and psychotic experiences: transnational data from the World Mental Health Survey. Br J Psychiatry. 2017;211(6):373-380.

26. Pignon B, Peyre H, Szöke A, et al. A latent class analysis of psychotic symptoms in the general population. Aust $N Z J$ Psychiatry. 2018;52(6):573-584.

27. Johns LC, Cannon M, Singleton N, et al. Prevalence and correlates of self-reported psychotic symptoms in the British population. Br J Psychiatry. 2004;185:298-305.

28. Kocsis-Bogár K, Miklósi M, Forintos DP. Impact of adverse life events on individuals with low and high schizotypy in a nonpatient sample. J Nerv Ment Dis. 2013;201(3):208-215.

29. Bardol O, Grot $\mathrm{S}, \mathrm{Oh} \mathrm{H}$, et al. Perceived ethnic discrimination as a risk factor for psychotic symptoms: a systematic review and meta-analysis. Psychol Med. 2020;50(7):1077-1089.

30. Kirkbride JB, Boydell J, Ploubidis GB, et al. Testing the association between the incidence of schizophrenia and social capital in an urban area. Psychol Med. 2008;38(8):1083-1094.

31. O'Donoghue B, Lyne JP, Renwick L, et al. Neighbourhood characteristics and the incidence of first-episode psychosis and duration of untreated psychosis. Psychol Med. 2016;46(7):1367-1378.

32. Solmi F, Colman I, Weeks M, Lewis G, Kirkbride JB. Trajectories of neighborhood cohesion in childhood, and psychotic and depressive symptoms at age 13 and 18 years. $J$ Am Acad Child Adolesc Psychiatry. 2017;56(7):570-577.

33. Lataster J, Myin-Germeys I, Lieb R, Wittchen HU, van Os J. Adversity and psychosis: a 10-year prospective study investigating synergism between early and recent adversity in psychosis. Acta Psychiatr Scand. 2012;125(5):388-399.

34. Gage SH, Jones HJ, Burgess S, et al. Assessing causality in associations between cannabis use and schizophrenia risk: a two-sample Mendelian randomization study. Psychol Med. 2017;47(5):971-980.

35. Colodro-Conde L, Couvy-Duchesne B, Whitfield JB, et al. Association between population density and genetic risk for schizophrenia. JAMA Psychiatry. 2018;75(9):901-910.

36. Gayer-Anderson C, Jongsma HE, Di Forti M, et al.; EU-GEI WP2 Group. The EUropean Network of National Schizophrenia Networks Studying Gene-Environment Interactions (EU-GEI): incidence and first-episode casecontrol programme. Soc Psychiatry Psychiatr Epidemiol. 2020;55(5):645-657. 
37. European Network of National Networks studying GeneEnvironment Interactions in Schizophrenia (EU-GEI). Identifying gene-environment interactions in schizophrenia: contemporary challenges for integrated, large-scale investigations. Schizophr Bull. 2014;40(4):729-736.

38. Jongsma HE, Gayer-Anderson C, Lasalvia A, et al.; European Network of National Schizophrenia Networks Studying GeneEnvironment Interactions Work Package 2 (EU-GEI WP2) Group. Treated incidence of psychotic disorders in the multinational EU-GEI study. JAMA Psychiatry. 2018;75(1):36- 46.

39. Stefanis NC, Hanssen M, Smirnis NK, et al. Evidence that three dimensions of psychosis have a distribution in the general population. Psychol Med. 2002;32(2):347-358.

40. Mark W, Toulopoulou T. Psychometric properties of "Community Assessment of Psychic Experiences": review and meta-analyses. Schizophr Bull. 2016;42(1):34-44.

41. Yung AR, Nelson B, Baker K, Buckby JA, Baksheev G, Cosgrave EM. Psychotic-like experiences in a community sample of adolescents: implications for the continuum model of psychosis and prediction of schizophrenia. Aust $N Z J$ Psychiatry. 2009;43(2):118-128.

42. Wigman JT, Vollebergh WA, Raaijmakers QA, et al. The structure of the extended psychosis phenotype in early adolescence - a cross-sample replication. Schizophr Bull. 2011;37(4):850-860.

43. Pignon B, Peyre H, Ferchiou A, et al.; EU-GEI WP2 Group Author. Assessing cross-national invariance of the Community Assessment of Psychic Experiences (CAPE). Psychol Med. 2019;49(15):2600-2607.

44. Bernstein DP, Stein JA, Newcomb MD, et al. Development and validation of a brief screening version of the Childhood Trauma Questionnaire. Child Abuse Negl. 2003;27(2):169-190.

45. Williams DR, Yu Y, Jackson JS, Anderson NB. Racial differences in physical and mental health: socio-economic status, stress and discrimination. J Health Psychol. 1997;2(3):335-351.

46. Jongsma HE, Gayer-Anderson C, Tarricone I, et al. Social disadvantage, linguistic distance, ethnic minority status and first-episode psychosis: results from the EU-GEI casecontrol study. Psychol Med. 2020;in press:1-13. https:// www.cambridge.org/core/journals/psychological-medicine/ article/social-disadvantage-linguistic-distance-ethnicminority-status-and-firstepisode-psychosis-resultsfrom-the-eugei-casecontrol-study/06D387C237DE2DAD354932B4E0575873

47. Hatch SL, Gazard B, Williams DR, Frissa S, Goodwin L, Hotopf M; SELCoH Study Team. Discrimination and common mental disorder among migrant and ethnic groups: findings from a South East London Community sample. Soc Psychiatry Psychiatr Epidemiol. 2016;51(5):689-701.

48. Lochner K, Kawachi I, Kennedy BP. Social capital: a guide to its measurement. Health Place. 1999;5(4):259-270.

49. Sampson RJ, Raudenbush SW, Earls F. Neighborhoods and violent crime: a multilevel study of collective efficacy. Science. 1997;277(5328):918-924.

50. Drukker M, Krabbendam L, Driessen G, van Os J. Social disadvantage and schizophrenia. A combined neighbourhood and individual-level analysis. Soc Psychiatry Psychiatr Epidemiol. 2006;41(8):595-604.

51. McCulloch A. An examination of social capital and social disorganisation in neighbourhoods in the British household panel study. Soc Sci Med. 2003;56(7):1425-1438.

52. Brugha T, Bebbington P, Tennant C, Hurry J. The List of Threatening Experiences: a subset of 12 life event categories with considerable long-term contextual threat. Psychol Med. 1985;15(1):189-194.

53. Motrico E, Moreno-Küstner B, de Dios Luna J, et al. Psychometric properties of the List of Threatening Experiences-LTE and its association with psychosocial factors and mental disorders according to different scoring methods. J Affect Disord. 2013;150(3):931-940.

54. Tortelli A, Nakamura A, Suprani F, et al. Subclinical psychosis in adult migrants and ethnic minorities: systematic review and meta-analysis. BJPsych Open. 2018;4(6):510-518.

55. Leaune E, Dealberto MJ, Luck D, et al. Ethnic minority position and migrant status as risk factors for psychotic symptoms in the general population: a meta-analysis. Psychol Med. 2019;49(4):545-558.

56. Murphy D, Vallières F, Murphy J, McElroy E, Hyland P. Risk factors associated with general and specific dimensions of psychosis in a nationally representative sample of adults from the United States. Psychosis. 2020;12(4):303-3013.

57. Kirkbride JB, Stochl J, Zimbrón J, et al. Social and spatial heterogeneity in psychosis proneness in a multilevel case-prodrome-control study. Acta Psychiatr Scand. 2015;132(4):283-292.

58. O'Donoghue B, Yung AR, Wood S, et al. Neighbourhood characteristics and the rate of identification of young people at ultra-high risk for psychosis. Schizophr Res. 2015;169(1-3):214-216.

59. Hunter DJ. Gene-environment interactions in human diseases. Nat Rev Genet. 2005;6(4):287-298.

60. Aas M, Pizzagalli DA, Laskemoen JF, et al. Elevated hair cortisol is associated with childhood maltreatment and cognitive impairment in schizophrenia and in bipolar disorders. Schizophr Res. 2019;213:65-71.

61. Reininghaus U, Kempton MJ, Valmaggia L, et al. Stress sensitivity, aberrant salience, and threat anticipation in early psychosis: an experience sampling study. Schizophr Bull. 2016;42(3):712-722.

62. Green JG, McLaughlin KA, Berglund PA, et al. Childhood adversities and adult psychiatric disorders in the national comorbidity survey replication I: associations with first onset of DSM-IV disorders. Arch Gen Psychiatry. 2010;67(2):113-123.

63. McLaughlin KA, Green JG, Gruber MJ, Sampson NA, Zaslavsky AM, Kessler RC. Childhood adversities and adult psychiatric disorders in the national comorbidity survey replication II: associations with persistence of DSM-IV disorders. Arch Gen Psychiatry. 2010;67(2):124-132.

64. Schürhoff F, Laguerre A, Fisher H, et al. Self-reported childhood trauma correlates with schizotypal measures in schizophrenia but not bipolar pedigrees. Psychol Med. 2009;39(3):365-370.

65. van Dam DS, van Nierop M, Viechtbauer W, et al.; Genetic Risk and Outcome of Psychosis (GROUP) investigators. Childhood abuse and neglect in relation to the presence and persistence of psychotic and depressive symptomatology. Psychol Med. 2015;45(7):1363-1377.

66. Janssen I, Hanssen M, Bak M, et al. Discrimination and delusional ideation. Br J Psychiatry. 2003;182:71-76.

67. Kong DT. Ethnic minorities' paranoia and self-preservative work behaviors in response to perceived ethnic discrimination, with collective self-esteem as a buffer. $J$ Occup Health Psychol. 2016;21(3):334-351.

68. Schmitt MT, Branscombe NR, Postmes T, Garcia A. The consequences of perceived discrimination for 
psychological well-being: a meta-analytic review. Psychol Bull. 2014;140(4):921-948.

69. Garety PA, Kuipers E, Fowler D, Freeman D, Bebbington PE. A cognitive model of the positive symptoms of psychosis. Psychol Med. 2001;31(2):189-195.

70. Myin-Germeys I, Delespaul P, van Os J. Behavioural sensitization to daily life stress in psychosis. Psychol Med. 2005;35(5):733-741.

71. Mortensen PB, Pedersen CB, Westergaard T, et al. Effects of family history and place and season of birth on the risk of schizophrenia. N Engl J Med. 1999;340(8):603-608.

72. Trotta A, Murray RM, Fisher HL. The impact of childhood adversity on the persistence of psychotic symptoms: a systematic review and meta-analysis. Psychol Med. 2015;45(12):2481-2498.

73. Polanczyk G, Moffitt TE, Arseneault L, et al. Etiological and clinical features of childhood psychotic symptoms: results from a birth cohort. Arch Gen Psychiatry. 2010;67(4): 328-338.

74. Binbay T, Drukker M, Elbi H, et al. Testing the psychosis continuum: differential impact of genetic and nongenetic risk factors and comorbid psychopathology across the entire spectrum of psychosis. Schizophr Bull. 2012;38(5):992-1002.

75. Sin J, Murrells T, Spain D, Norman I, Henderson C. Wellbeing, mental health knowledge and caregiving experiences of siblings of people with psychosis, compared to their peers and parents: an exploratory study. Soc Psychiatry Psychiatr Epidemiol. 2016;51(9):1247-1255.

76. van Dam DS, Korver-Nieberg N, Velthorst E, Meijer CJ, de Haan L; For Genetic Risk and Outcome in Psychosis (GROUP). Childhood maltreatment, adult attachment and psychotic symptomatology: a study in patients, siblings and controls. Soc Psychiatry Psychiatr Epidemiol. 2014;49(11):1759-1767.
77. van Os J, Hanssen M, Bak M, Bijl RV, Vollebergh W. Do urbanicity and familial liability coparticipate in causing psychosis? Am J Psychiatry. 2003;160(3):477-482.

78. Baldwin JR, Reuben A, Newbury JB, Danese A. Agreement between prospective and retrospective measures of childhood maltreatment: a systematic review and meta-analysis. $J A M A$ Psychiatry. 2019;76(6):584-593.

79. MacDonald K, Thomas ML, MacDonald TM, Sciolla AF. A perfect childhood? Clinical correlates of minimization and denial on the childhood trauma questionnaire. $J$ Interpers Violence. 2015;30(6):988-1009.

80. Dohrenwend BP. Inventorying stressful life events as risk factors for psychopathology: toward resolution of the problem of intracategory variability. Psychol Bull. 2006;132(3):477-495.

81. Oh H, Yang LH, Anglin DM, DeVylder JE. Perceived discrimination and psychotic experiences across multiple ethnic groups in the United States. Schizophr Res. 2014;157(1-3):259-265.

82. Oh H, Cogburn CD, Anglin D, Lukens E, DeVylder J. Major discriminatory events and risk for psychotic experiences among Black Americans. Am J Orthopsychiatry. 2016;86(3):277-285.

83. Rakhshan Rouhakhtar PJ, Pitts SC, Schiffman J. Associations between Race, discrimination, community violence, traumatic life events, and psychosis-like experiences in a sample of college students. J Clin Med. 2019;8(10).

84. Di Forti M, Quattrone D, Freeman TP, et al.; EU-GEI WP2 Group. The contribution of cannabis use to variation in the incidence of psychotic disorder across Europe (EU-GEI): a multicentre case-control study. Lancet Psychiatry. 2019;6(5):427-436.

85. Vassos E, Pedersen CB, Murray RM, Collier DA, Lewis CM. Meta-analysis of the association of urbanicity with schizophrenia. Schizophr Bull. 2012;38(6):1118-1123. 